\title{
Differential Cytokine Expression Analyses of CEFs and DEFs Infected with H6 Influenza Virus Strains Isolated from Anseriformes in Anhui Province, China
}

\author{
Ye Ge ${ }^{1,2,3}$, Qiucheng $\mathrm{Yao}^{1}$, Hongliang $\mathrm{Chai}^{3}$, Yuping $\mathrm{Hua}^{3 *}$ and Guohua Deng ${ }^{2 *}$ \\ ${ }^{I}$ College of Coastal Agricultural Sciences, Guangdong Ocean University, \\ Zhanjiang, Guangdong Province, China \\ ${ }^{2}$ State Key Laboratory of Veterinary Biotechnology, Harbin Veterinary Research \\ Institute, Chinese Academy of Agricultural Sciences, Harbin, China \\ ${ }^{3}$ College of Wildlife Resources, Northeast Forestry University, Harbin, Heilongjiang \\ Province, China
}

\begin{abstract}
A B S T R A C T
Wild birds, as reservoirs of viruses, play active roles in influenza virus transmission and reassortment. To further evaluate the potential infection risk of H6 AIVs from wild birds, we inoculated these viruses into A549 cells, which showed that these wild bird H6 AIVs have acquired the ability to cross interspecific barriers and have the potential to infect mammals. We analysed the level of cytokine expression in response to four H6 AIVs in CEFs and DEFs using real-time PCR. The results showed that the viruses from wild birds could induce a higher level of cytokine expression in CEFs than in DEFs. These findings suggested that H6 AIV in wild birds, especially migratory species, play an indispensable role in the spread and reassortment of AIVs and provide unknown opportunities for mutation and emergence of novel influenza viruses. Additional studies of AIVs originating from wild birds will help to determine viral adaptation and maintenance in alternative hosts.
\end{abstract}

\begin{tabular}{l} 
Article Information \\
\hline Received 01 July 2019 \\
Revised 11 September 2019 \\
Accepted 20 September 2019 \\
Available online 11 January 2021 \\
Authors' Contribution \\
\hline YG and QY conducted the \\
experiments. YG drafted the \\
manuscript while HC and HY \\
revised it. GD and HY supported in \\
experiments. GD supplied funds for \\
the study. \\
Key words \\
Chicken embryo fibroblasts, Duck \\
embryo fibroblasts, Influenza virus, \\
Bean goose, H6 subtype, Cytokine \\
expression, PCR
\end{tabular}

expression, PCR

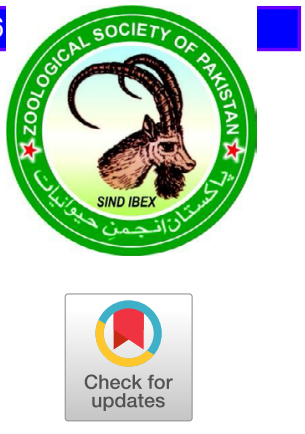

\section{INTRODUCTION}

$\mathrm{W}$ ild birds represent major natural reservoirs for avian influenza viruses (AIVs), which have been associated with stable host switch events to novel hosts, including domestic gallinaceous poultry, horses, swine, and humans, leading to the emergence of influenza A virus lineages that are transmissible in the new host (Olsen et al., 2006). In recent years, different subtypes of influenza viruses (such as $\mathrm{H} 5, \mathrm{H} 6, \mathrm{H} 7$, and $\mathrm{H} 10$ ), which are pathogenic to humans, have emerged in poultry in succession, crossing the species barrier and leading to frequent outbreaks of human infection (Liu et al., 2013).

H6 AIV was first discovered in turkeys in Massachusetts, USA, in 1965 (Wang et al., 2014). Currently, H6 AIVs are widely distributed and have been detected in different species of animals worldwide $(\mathrm{Wu}$ et al., 2015). The biological characteristics of $\mathrm{H} 6$ viruses indicate that this subtype can have a profound impact on

\footnotetext{
* Corresponding author: dgh1971@163.com; yuping_ hua@126.com

0030-9923/2021/0002-0447 \$ 9.00/0

Copyright 2021 Zoological Society of Pakistan
}

human health. On June 21, 2013, the first case of a human infection by an avian-origin H6N1 influenza A virus (A/ Taiwan/2/2013, Taiwan2) was reported by the Taiwan Centres for Disease Control (Shi et al., 2013). Human infection with $\mathrm{H} 6$ in mainland China has not been reported, but a serum antibody positive for the $\mathrm{H} 6$ virus has been found in poultry workers. More than $30 \%$ of the H6 AIVs from poultry circulating in China have enhanced affinity to human-like receptors (a-2, 6 NeuAcGal) (Wang et al., 2014). Some strains of the H6 subtype can also infect mice without prior adaptation (Chen et al., 2009), and some can be transmitted efficiently among guinea pigs (Wang et al., 2014). These events are an indication that H6 AIVs can cross species barriers and directly infect humans. As the natural host of AIVs, wild birds play an important role in virus reassortment, transmission and even cross-species transmission (Fouchier and Munster, 2009). However, existing research on the biological assessment and potential threat to mammals of H6 AIVs from wild birds is less prevalent than that conducted on poultry.

\footnotetext{
Abbreviations

AIVs, avian influenza viruses; CEFs, chicken embryo fibroblasts; DEFs, duck embryo fibroblasts; HPAV, highly pathogenic avian virus.
} 
In infected duck, highly pathogenic avian virus (HPAV) is asymptomatic, rarely causes death, and only evokes a weak immune response (Philpott et al., 1989). However, in infected chicken, HPAV evokes a strong immune response and triggers a "cytokine storm" (Suarez and Schultz-Cherry, 2000). The host has been shown to play an important role in AIV infection. Many studies have reported that the host innate immune response significantly functions in resisting AIV infection (Higgins et al., 1987; Wang et al., 2015). However, no immediate reports about the immune responses of chicken and duck to AIVs from wild birds are available.

In this study, two strains of the H6 virus, H6N1 and H6N2, which showed good proliferative abilities in mice, were selected for infection experiments in A549 cells. Both viruses could grow in A549 cells, indicating that H6 AIVs have the potential to infect humans. Because of frequent contact between wild birds and poultry, virus originating in wild birds may be transmitted to poultry or undergo reassortment between poultry and wild birds and then cause spreading. Comparison of cytokine expression in chicken embryo fibroblasts (CEFs) with that in duck embryo fibroblasts (DEFs) revealed that these viruses evoked higher cytokine expression levels in CEFs than in DEFs. H6 AIVs have received increasing attention from researchers. As natural hosts, wild birds increase the scale of influenza virus during their seasonal migration and accelerate viral gene reassortment. The surveillance of wild birds for early warning, prevention, and control of viral outbreaks should be enhanced to reduce the risk of pathogen emergence from wildlife host reservoirs.

\section{MATERIALS AND METHODS}

\section{Viral replication in human lung cells (A549)}

A549 cell (human alveolar epithelial cell) monolayers were infected at a multiplicity of infection (MOI) of 0.01 . The cells were incubated at $37^{\circ} \mathrm{C}$ in Opti-MEM (Gibco, Thermo Fisher, Australia) containing $0.2 \mu \mathrm{g} / \mathrm{ml}$ TPCKtreated trypsin (TPCK-treated trypsin, Sigma, USA). Culture supernatant containing viruses was collected at five time points (hours post infection) and titrated in embryonated chicken eggs. The growth data shown are averages of the results of three independent experiments.

\section{Virus, cells and cell infection}

We prepared CEFs andDEFs according to conventional methods (Liang et al., 2011). In brief, 10-12-day-old SPF chicken embryos and SPF duck embryos were obtained from Harbin Veterinary Research Institute (HVRI) of the Chinese Academy of Agricultural Sciences (CAAS). The cells were cultured overnight in $8 \%$ foetal bovine serum (FBS)/DMEM with a density of $1 \times 10^{6}$ cells per plate.
After the cells were cultured overnight, the cells were washed with sterile PBS to remove the adherent cells. The cells were infected with $100 \mu$ l of virus at a MOI of 0.01 . Culture plates were rocked 4 times during incubation for 1 h. One hour later, the cells were washed with sterile PBS three times to remove the viruses completely. The media were replaced with $2 \mathrm{ml}$ of Opti-MEM supplemented with $1 \mu \mathrm{g} / \mu 1$ TPCK trypsin per plate. The cell monolayer was collected at the indicated time points $(2,4,8,12,24$ and 36 $\mathrm{h}$ post-infection), and RNA was extracted.

\section{$R N A$ extraction and DNA preparation}

RNA was extracted according to the TRIzol regent extraction protocol (Invitrogen). RNA samples were quantified using a spectrophotometer. RNA samples were treated with a PrimeScript ${ }^{\mathrm{TM}}$ RT Reagent Kit with gDNA Eraser (TaKaRa, Japan) before use as a template for cDNA synthesis. Transcription was performed using a TaKaRa One Step Kit with SYBR® Premix Ex Taq ${ }^{\mathrm{TM}}$ II (TaKaRa, Japan) in a $40 \mu \mathrm{l}$ volume containing $1 \mu \mathrm{g}$ RNA.

\section{Quantitative real-time PCR}

qRT-PCR was performed using the specific primers designed by Primer Premier 5.0 and Oligo 7.0 software based on published and previously reported target sequences (Adams et al., 2009) (Table I). We verified the primers' specificity by sequencing and dissociation curves. The expression levels of cytokines were monitored on an Agilent Mx3005p using SYBR Premix Ex Taq ${ }^{\mathrm{TM}}$ II (TaKaRa). The reaction conditions were as follows: $95^{\circ} \mathrm{C}$ for $\sim 30 \mathrm{~s}$, followed by 40 cycles of $95^{\circ} \mathrm{C}$ for $\sim 5 \mathrm{~s}, 56 \sim 60^{\circ} \mathrm{C}$ for $30 \mathrm{~s}$ and $72^{\circ} \mathrm{C}$ for $\sim 30 \mathrm{~s}$. Dissociation curves of the fluorescent quantitative PCR products were generated at the final step.

\section{Calculations and statistics}

We normalize the expression levels of these cytokines using the housekeeping gene glyceraldehyde-3-phosphatedehydrogenase (GAPDH) using the $-{ }^{\triangle} \mathrm{Ct}$ method (Li et al. 2006). Every time point was compared against the baseline (at $0 \mathrm{~h}$ ). The data were analysed by the fold change. The error bar was calculated by three replicates for each cytokine measured.

\section{RESULTS}

\section{Replication of H6 viruses in A549 cells}

We selected two H6 viruses (one N1 subtype and one N2 subtype) based on their NA subtypes and proliferation ability in mice. The two viruses were inoculated in A549 monolayers at a MOI of 0.01 . Then, the cell culture supernatants were collected after 12, 24, 48, 60, and 72 h. The two H6 viruses in this study replicated well in 
Table I. Primers used in quantitative RT-PCR.

\begin{tabular}{|c|c|c|}
\hline Name of primer & Sequence & GenBank number \\
\hline DIL-6 F & TTCGACGAGGAGAAATGCTT & AB191038 \\
\hline DIL-6 R & CCTTATCGTCGTTGCCAGAT & \\
\hline DIL-8 F & AGGACAACAGAGAGGTGTGCTTG & NM_205498 \\
\hline DIL-8 R & GCCTTTACGATCCGCTGTACC & \\
\hline DIL-10 F & GGGGAGAGGAAACTGAGAGATG & JN786941.1 \\
\hline DIL-10 R & TCACTGGAGGGTAAAATGCAGA & \\
\hline DTLR7 F & CCTTTCCCAGAGAGCATTCA & AY940195 \\
\hline DTLR7 R & TCAAGAAATATCAAGATAATCACATCA & \\
\hline DIFN- $\alpha \mathrm{F}$ & TTGCTCCTTCCCGGACA & EF053034 \\
\hline DIFN- $\alpha \mathrm{R}$ & GCTGAGGGTGTCGAAGAGGT & \\
\hline DGAPDHF & ATGTTCGTGATGGGTGTGAA & AY436595 \\
\hline DGAPDHR & CTGTCTTCGTGTGTGGCTGT & \\
\hline$(\mathrm{C}$ and $\mathrm{D}) \mathrm{IFN}-\beta \mathrm{F}$ & AGATGGCTCCCAGCTCTACA & AY831397 \\
\hline$(\mathrm{C}$ and $\mathrm{D}) \mathrm{IFN}-\beta \mathrm{R}$ & AGTGGTTGAGCTGGTTGAGG & \\
\hline CIL-6 F & ATGTGCAAGAAGTTCACCGTG & EU170468 \\
\hline CIL-6R & TTCCAGGTAGGTCTGAAAGGCGAA & \\
\hline CIL-8F & AAGTTCATCCACCCTAAATC & NM_205498 \\
\hline CIL-8R & GCATCAGAATTGAGCTGAGC & \\
\hline CIL-10 F & TGCTGCGCTTCTACACAGAT & NC_006113 \\
\hline CIL-10 R & TGGCTTTGCTCCTCTTCTCG & \\
\hline CTLR-7F & TGTGATGTGGAAGCCTTTGA & DQ780342 \\
\hline CTLR-7R & ATTATCTTTGGGCCCCAGTC & \\
\hline CIFN- $\alpha \mathrm{F}$ & CAACGACACCATCCTGGACA & EU367971 \\
\hline CIFN- $\alpha \mathrm{R}$ & ATCCGGTTGAGGAGGCTTTG & \\
\hline CGAPDHF & CCTCTCTGGCAAAGTCCAAG & V00407 \\
\hline CGAPDHR & CATCTGCCCATTTGATGTTG & \\
\hline
\end{tabular}

A549 cells, suggesting that H6 subtype AIVs could be a threat to humans (Fig. 1).

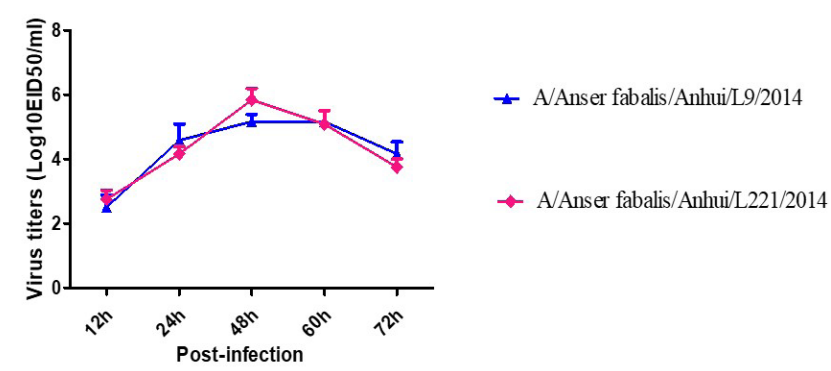

Fig. 1. Multi-cycle replication of H6 avian influenza viruses in A549 cells. A549 monolayers were inoculated with virus at a MOI of 0.01 , and the culture supernatants were collected at the indicated time points and then titrated in eggs.
Analysis of the expression of pro-inflammatory factors, antiviral cytokines, an anti-inflammatory cytokine and a pathogen recognition receptor in CEFs and DEFs

To understand the function of the innate immune response in the good replication of AIVs in mice, we compared the expression levels of cytokines, including proinflammatory factors (IL-6 and IL-8), antiviral cytokines (IFN- $\alpha$ and IFN- $\beta$ ), an anti-inflammatory cytokine (IL-10) and a pathogen recognition receptor (TLR7). The expression levels of IL-6 were similar in CEFs and DEFs separately infected with the four virus strains, with low expression and obvious down-regulation. Only in the early stage $(2 \mathrm{~h}$ post-infection) did the viruses evoke a slightly high level of IL-6 expression in CEFs. In DEFs, IL- 6 expression levels did not cross the baseline at any time point. The expression levels of IL-6 did not significantly differ in CEFs and DEFs infected with any of the virus strains; the same result was observed for each virus strain (Fig. 2A). 

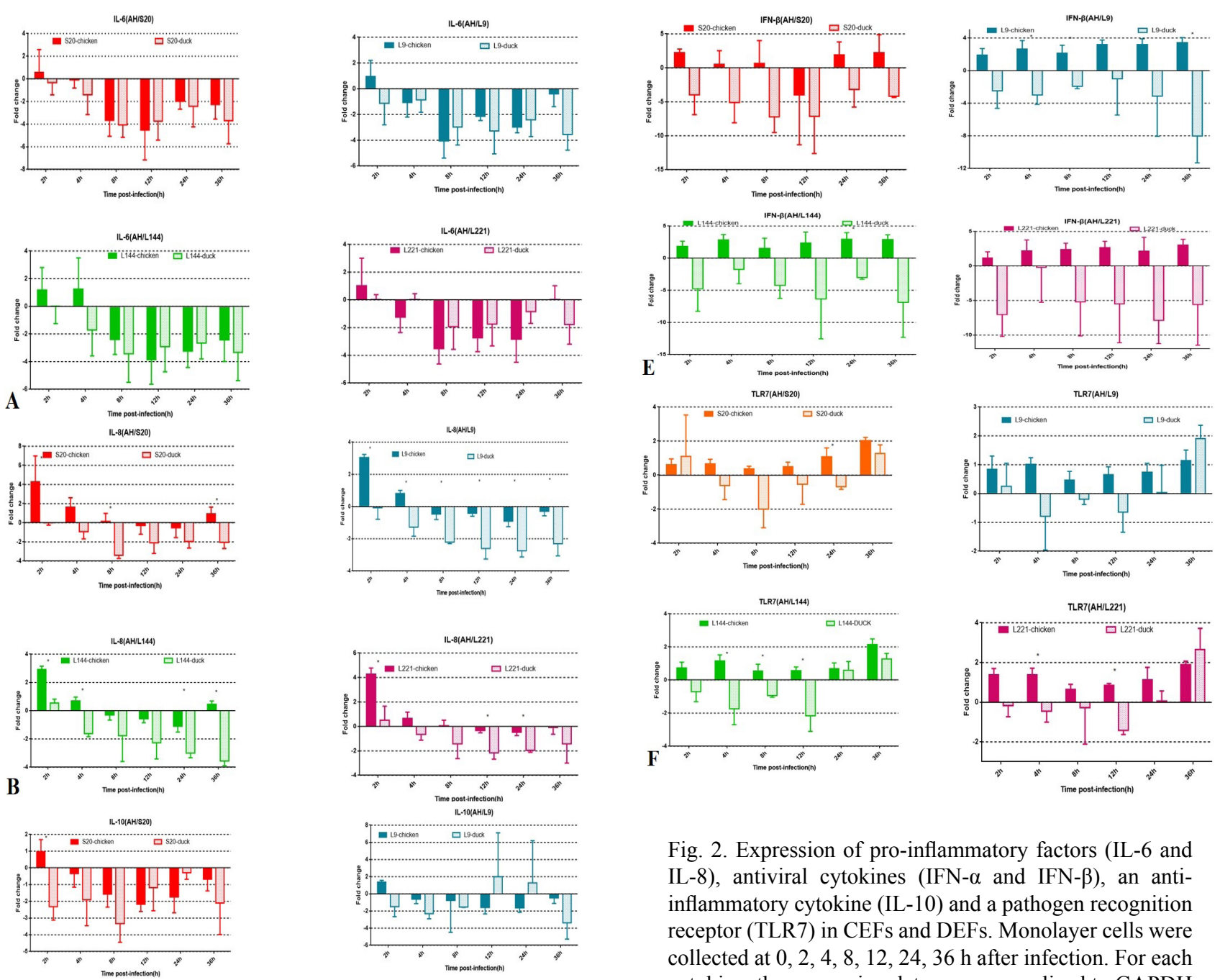

Fig. 2. Expression of pro-inflammatory factors (IL-6 and IL-8), antiviral cytokines (IFN- $\alpha$ and IFN- $\beta$ ), an antiinflammatory cytokine (IL-10) and a pathogen recognition receptor (TLR7) in CEFs and DEFs. Monolayer cells were collected at $0,2,4,8,12,24,36 \mathrm{~h}$ after infection. For each cytokine, the expression data were normalized to GAPDH expression. The column data were calculated by $-\triangle \triangle \mathrm{Ct}$. The results are presented as the mean fold change \pm SD Error bars represent standard error.

The levels of IL-8 expression in CEFs and DEFs showed obvious differences. In CEFs, the level of IL-8 expression was higher than that in DEFs. Over time, the level of IL-8 expression declined gradually. At $36 \mathrm{~h}$, the expression level showed a slight recovery, except for cells infected with $\mathrm{AH} / \mathrm{L} 144$. Of the CEFs infected with these four viruses, IL-8 expression levels were obviously higher in CEFs infected with $\mathrm{AH} / \mathrm{S} 20$ and $\mathrm{AH} / \mathrm{L} 221$ than in CEFs infected with AH/L9 and AH/L144. Similar to IL-6 expression, a higher fold change in IL-8 expression was observed in CEFs than in DEFs (Fig. 2B).

Regarding type I IFN, we compared IFN-a and IFN- $\beta$ expression. The fold changes in their expression levels were much larger, with approximately ten-fold differences and exited significant difference between 
CEFs and DEFs in IFN-a expression In CEFs, IFN-a and IFN- $\beta$ expression reached the highest levels at $36 \mathrm{~h}$ postinfection. In DEFs, the sign of significant difference was not existing in all four viruses except only $\mathrm{AH} / \mathrm{L} 9$ at $4 \mathrm{~h}$ and $36 \mathrm{~h}$ post-infection (Fig. 2D and 2E). IFN-a and IFN- $\beta$ expression results indicated that chicken could trigger a strong immune response to antivirus but that duck may not. IL-10 expression appeared at $2 \mathrm{~h}$ post-infection and then decreased over time. Most time points showed downregulation. Remarkably, AH/L9 infected DEFs, with high expression at 12 and $24 \mathrm{~h}$ (Fig. 2D).

We next compared the differences in TLR 7 expression levels in CEFs and DEFs. Once again, higher levels were observed in CEFs than in DEFs. TLR 7 expression reached the highest level at $36 \mathrm{~h}$ in both CEFs and DEFs (Fig. 2F).

\section{DISCUSSION}

According to the morphological characteristics of the birds and the faeces collected, the bird host of these H6 AIVs was the bean goose, which taxonomically belongs to the Anseriformes family. When these H6 viruses were infected into DEFs and CEFs, a much higher titre of viral amplification was found in DEFs than in CEFs after 48 $\mathrm{h}$ (data not shown). This phenomenon of cell preference further confirmed that waterfowl were the hosts of these H6 viruses.

Poultry is a basilica linkage between wild birds and humans in the emergence and duration of virus, which could potentially infect humans. CEFs and DEFs can function as initial research models when studying influenza virus infection to detect the host immune response in poultry. We compared the differences in the expression of cytokines, including pro-inflammatory factors, antiviral cytokines, an anti-inflammatory cytokine and a pathogen recognition receptor to further understand whether these viruses evoked immune responses and whether the pathogenesis of these viruses differed between the two important host species of influenza virus to preliminary evaluate the transmission of these viruses from wild birds to poultry. IL-6 may have an important role in mediating clinical symptoms after AIV infection (Liang et al., 2011; Skoner et al., 1999). In this study, the expression level of IL-6 was down-regulated in cells infected with virus. The animal experiments confirmed this result; all of the mice in the infected groups had no clinical symptoms. NS1 protein has a significant function in inducing type I IFN. In our study, the expression levels of IFN- $\alpha$ and IFN- $\beta$ remarkably differed, thus indicating differing susceptibilities of CEFs and DEFs to the inhibitory action of the NS1 gene on type I IFN expression (Goraya et al., 2017). Recognized as an anti-inflammatory cytokine, IL-10 could modulate many cell types to regulate immune and inflammatory responses (Moore et al., 2001; Zou et al., 2014).

H6 AIVs are distributed worldwide, similar to H5N1 and H9N2 (Pepin et al., 2013) and cause considerable losses in poultry farming. The case of an H6-infected human serves as a warning. As the natural host of influenza virus, wild birds, especially migratory species, play an indispensable role in the spread and reassortment of AIVs and provide unknown opportunities for mutation and emergence of novel influenza viruses (Driskell et al., 2010). Additional studies of AIVs originating from wild birds will help to determine viral adaptation and maintenance in alternative hosts.

\section{ACKNOWLEDGMENTS}

This research was supported by the 13th FiveYear National Key Research Project which grant No. 2016YFD0500201, "Fundamental Research Funds for the Central Universities" (2572017CA19) and the 13th FiveYear National Key Research Project: The Migration of Wild Animal Epidemic Disease Transmission Risk Research Project (Grant No. 2016YFC1201600). We also thank the State Key Laboratory of Veterinary Biotechnology, Harbin Veterinary Research Institute, Chinese Academy of Agricultural Sciences. We are also grateful to some other related laboratories for their technical support.

\section{Statement of conflict of interest}

None of the authors have a conflict of interest.

\section{REFFERENCES}

Adams, S.C., Xing, Z., Li, J. and Cardona, C.J., 2009. Immune-related gene expression in response to H11N9 low pathogenic avian influenza virus infection in chicken and Pekin duck peripheral blood mononuclear cells. Mol. Immunol., 46:1744-1749. https://doi.org/10.1016/j.molimm.2009.01.025

Chen, Z., Santos, C., Aspelund, A., Gillim-Ross, L., Jin, H., Kemble, G. and Subbarao, K., 2009. Evaluation of live attenuated influenza a virus $\mathrm{h} 6$ vaccines in mice and ferrets. J. Virol., 83: 65-72. https://doi. org/10.1128/JVI.01775-08

Driskell, E.A., Jones, C.A., Stallknecht, D.E., Howerth, E.W. and Tompkins, S.M., 2010. Avian influenza virus isolates from wild birds replicate and cause disease in a mouse model of infection. Virology, 399: 280-289. https://doi.org/10.1016/j. virol.2010.01.005

Fouchier, R.A. and Munster, V.J., 2009. Epidemiology of low pathogenic avian influenza viruses in 
wild birds. Rev. Sci. Tech., 28: 49-58. https://doi. org/10.20506/rst.28.1.1863

Goraya, M.U., Ali, L. and Younis, I., 2017. Innate immune responses against avian respiratory viruses. Hosts Viruses, 5: 78-87. http://dx.doi.org/10.17582/ journal.hv/2017/4.5.78.87

Higgins, D.A., Shortridge, K.F. and Ng, P.L., 1987. Bile immunoglobulin of the duck (Anas platyrhynchos). II. Antibody response in influenza A virus infections. Immunology, 62: 499-504.

Li, M.O., Wan, Y.Y., Sanjabi, S., Robertson, A.K. and Flavell, R.A., 2006. Transforming growth factorbeta regulation of immune responses. Annu. Rev. Immunol., 24: 99-146. https://doi.org/10.1146/ annurev.immunol.24.021605.090737

Liang, Q.L., Luo, J., Zhou, K., Dong, J.X. and He, H.X., 2011. Immune-related gene expression in response to $\mathrm{H} 5 \mathrm{~N} 1$ avian influenza virus infection in chicken and duck embryonic fibroblasts. Mol. Immunol., 48: 924-930. https://doi.org/10.1016/j. molimm.2010.12.011

Liu, D., Shi, W., Shi, Y., Wang, D., Xiao, H., Li, W., Bi, Y., Wu, Y., Li, X., Yan, J., Liu, W., Zhao, G., Yang, W., Wang, Y., Ma, J., Shu, Y., Lei, F. and Gao, G.F., 2013. Origin and diversity of novel avian influenza A H7N9 viruses causing human infection: phylogenetic, structural and coalescent analyses. Lancet, 381: 1926-1932. https://doi.org/10.1016/ S0140-6736(13)60938-1

Moore, K.W., Malefyt, R.W., Coffman, R.L. and O'Garra, A., 2001. Interleukin-10 and the interleukin-10 receptor. Annu. Rev. Immunol., 19: 683-765. https://doi.org/10.1146/annurev. immunol.19.1.683

Olsen, B., Munster, V.J., Wallensten, A., Waldenstrom, J., Osterhaus, A.D. and Fouchier, R.A., 2006. Global patterns of influenza a virus in wild birds. Science, 312: 384-388. https://doi.org/10.1126/ science. 1122438

Pepin, K.M., Wang, J., Webb, C.T., Smith, G.J., Poss, M., Hudson, P.J., Hong, W., Zhu, H., Riley, S. and Guan, Y., 2013. Multiannual patterns of influenza A transmission in Chinese live bird market systems. Influenza Other Respir. Viruses, 7: 97-107. https:// doi.org/10.1111/j.1750-2659.2012.00354.x

Philpott, M.S., Easterday, B.C. and Hinshaw, V.S., 1989. Antigenic and phenotypic variants of a virulent avian influenza virus selected during replication in ducks. J. Wildl. Dis., 25: 507-513. https://doi. org/10.7589/0090-3558-25.4.507

Shi, W., Shi, Y., Wu, Y., Liu, D. and Gao, G.F., 2013. Origin and molecular characterization of the human-infecting H6N1 influenza virus in Taiwan. Protein Cell, 4: 846-853. https://doi.org/10.1007/ s13238-013-3083-0

Skoner, D.P., Gentile, D.A., Patel, A. and Doyle, W.J., 1999. Evidence for cytokine mediation of disease expression in adults experimentally infected with influenza A virus. J. Infect. Dis., 180:10-4. https:// doi.org/10.1086/314823

Suarez, D.L. and Schultz-Cherry, S., 2000. Immunology of avian influenza virus: A review. Dev. Comp. Immunol., 24: 269-283. https://doi.org/10.1016/ S0145-305X(99)00078-6

Wang, G., Deng, G., Shi, J., Luo, W., Zhang, G., Zhang, Q., Liu, L., Jiang, Y., Li, C., Sriwilaijaroen, N., Hiramatsu, H., Suzuki, Y., Kawaoka, Y. and Chen, H., 2014. H6 influenza viruses pose a potential threat to human health. J. Virol., 88: 3953-3964. https://doi.org/10.1128/JVI.03292-13

Wang, J., Tang, C., Wang, Q., Li, R., Chen, Z., Han, X., Wang, J. and $\mathrm{Xu}, \mathrm{X} ., 2015$. Apoptosis induction and release of inflammatory cytokines in the oviduct of egg-laying hens experimentally infected with H9N2 avian influenza virus. Vet. Microbiol., 177: 302314. https://doi.org/10.1016/j.vetmic.2015.04.005

Wu, H., Peng, X., Peng, X., Cheng, L. and Wu, N., 2015. Molecular characterization of novel reassortant H6N2 subtype avian influenza viruses isolated from poultry in Eastern China, in 2014. Infect. Genet. Evol., 36: 41-45. https://doi.org/10.1016/j. meegid.2015.08.043

Zou, Q., Wu, B., Xue, J., Fan, X., Feng, C., Geng, S., Wang, M. and Wang, B., 2014. CD8+ Treg cells suppress CD8+ T cell-responses by IL-10dependent mechanism during H5N1 influenza virus infection. Eur. J. Immunol., 44: 103-114. https:// doi.org/10.1002/eji.201343583 